\title{
Raoultella Planticola; A Rare Case of Urinary Tract Infection in a Three-Year-Old Healthy Female
}

\author{
Mariyam Hashmi ${ }^{1}$ and Myra Chiang ${ }^{2 *}$ \\ ${ }^{1}$ Department of Pediatrics, Charleston Area Medical Center/WVU-Charleston Division, USA \\ ${ }^{2}$ Department of Pediatric Nephrology, West Virginia University, Charleston Division, USA
}

*Corresponding author: Myra Chiang, Department of Pediatric Nephrology, West Virginia University, Charleston Division, USA

\begin{tabular}{|c|c|}
\hline ARTICLE INFO & ABSTRACT \\
\hline & Abbreviations: UTI: Urinary Tract Infection; IgA: Immunoglobulin A; SLE: Systemic \\
\hline Published: 蔧 February 21, 2020 & Lupus Erythematosus; SG: Specific Gravity; LE: Leukocyte Esterase \\
\hline
\end{tabular}

Citation: Mariyam Hashmi, Myra Chiang. Raoultella Planticola; A Rare Case of Urinary Tract Infection in a Three-Year-Old Healthy Female. Biomed J Sci \& Tech Res 25(5)-2020. BJSTR. MS.ID.004270.

\section{Introduction}

Raoultella planticola is a gram-negative, aerobic, non-motile bacterium found mostly in the environment. There is a high prevalence in both the soil and in water [1]. There are only a handful of case reports of Raoultella planticola causing symptomatic infections in human beings [2]. Most of the reported infections occurred in immunocompromised adult patients with multiple co-morbidities. Infection in immunocompromised patients is associated with a poor prognosis [3]. Of the available literature reviewed, there are only two cases of Raoultella planticola causing a symptomatic Urinary Tract Infection (UTI) in pediatric patients. The first was a case of a 16-month-old male with a history of rhabdomyosarcoma of the bladder neck and cystitis and the other a UTI in otherwise healthy 2-month-old female $[2,4]$. We are presenting a case of a healthy three-year-old female with a symptomatic UTI caused by Raoultella planticola.

Our patient is a three-year-old previously healthy caucasian female who presents with recurrent episodes of poorly localized abdominal and back pain for six months. Patient's mother reports that our patient initially complained of abdominal pain daily for one month along with some episodes of dysuria. She did not have any fevers, hematuria or other urinary complaints. There is no history of constipation and she does not take any medication. She is fully potty-trained and does not wear diapers. She had an unremarkable birth history; she was born at term via spontaneous vaginal delivery without any complications. She is up to date with her immunizations and is developmentally appropriate for her age. Our patient's mother has nephrolithiasis and her second cousin has Immunoglobulin A (IgA) Nephropathy. Family history is also positive for her maternal great grandmother with Systemic Lupus Erythematosus (SLE). One week into her symptom onset, her primary care physician ordered a urine dipstick which showed a urine Specific Gravity (SG) 1.010, 2+ Leukocyte Esterase (LE), trace ketones, nitrites, protein, glucose and blood were all negative. She was treated with a ten-day course of trimethoprim-sulfamethoxazole. Mom reports that our patient's abdominal pain persisted into week two despite treatment, repeat urine dipstick was obtained, which now showed a SG 1.025, pH 6.0, trace LE, nitrites, protein, glucose, blood were all negative. During this period our patient had two episodes of vulvovaginitis that were treated with topical miconazole nitrate.

Her intermittent abdominal and back pain persisted so another urine dipstick was obtained four months into symptom onset at that time SG 1.010, pH 6.5, small LE, nitrite positive, with trace blood, protein and glucose were negative. Urine culture was positive for Raoultella planticola $(>100,000 \mathrm{cfu} / \mathrm{ml})$, it was sensitive to trimethoprim-sulfamethoxazole, with resistance only to Ampicillin. 
She was once again treated with trimethoprim-sulfamethoxazole for ten days with resolution of symptoms. Renal ultrasound was obtained after the infection resolved which was unremarkable apart from some mild dilatation of her renal pelvis bilaterally. Abdominal x-ray showed some stool but no signs of obstruction. On presentation to our clinic her blood pressure was 115/57 $\mathrm{mmHg}$, heart rate 113 beats per minute, her height $101 \mathrm{~cm}$ and weight is $17.9 \mathrm{~kg}$, BMI 17.55 ( $91^{\text {st }}$ percentile for age). Physical exam was unremarkable apart from allergic shiners. Genital exam was appropriate for age, without any labial adhesions with some mild whitish discharge. We repeated a urine dipstick which showed a SG $>1.030$, pH 5.5, small LE, nitrite neg, trace blood, with no protein, glucose or ketones. Urine culture was negative.

\section{Discussion}

Raoultella planticola is a non-motile, aerobic, encapsulated gram-negative rod often difficult to distinguish from Klebsiella [1]. It was first described as Klebsiella planticola and Klebsiella trevisanii in the 1980s [5]. It has been found in human body fluids including saliva, urine, and stool. There is a limited amount of data available regarding this pathogen and its effects in human beings, especially in those that are immunocompetent, specifically healthy pediatric patients. There does not seem be an association with poor prognosis in healthy patients. Infection with Raoultella planticola theoretically can affect any organ system in the body but in the pediatric cases reviewed, it predominately affects the urinary tract. The rarity of infection with this organism is the reason that the pathogenesis is not understood well. It is also alarming that human infections with Raoultella planticola are on the rise [3]. Therefore,

ISSN: 2574-1241

DOI: $10.26717 /$ BJSTR.2020.25.004270

Myra Chiang. Biomed J Sci \& Tech Res

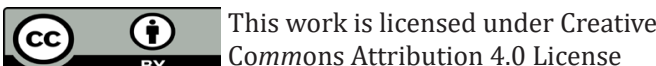

Submission Link: https://biomedres.us/submit-manuscript.php there is a need for further research highlighting the infectivity patterns and outcomes associated with this organism. The mainstay treatment of Raoultella planticola infection of the urinary tract is broad-spectrum antibiotics with gram-negative coverage. The strain of Raoultella planticola our patient grew was resistant to Ampicillin though resistance patterns are not often reported for this organism.

\section{Conclusion}

Raoultella planticola is a rare cause of UTI in healthy pediatric patients and there is limited literature available on the pathogenesis of the organism. It is important to be aware of this organism and its potential as an emerging pathogen. It is also of the utmost importance to closely monitor its patterns of antibiotic resistance.

\section{References}

1. Mehmood H, Pervin N, Israr Ul Haq M, Kamal KR, Marwat A, et al. (2018) A Rare Case of Raoultella Planticola Urinary Tract Infection in a Patient with Immunoglobulin A Nephropathy. J Investigative Med High Impact Case Rep 6: 2324709618780422.

2. Howell C, Fakhoury J (2017) A Case of Raoultella Planticola Causing a Urinary Tract Infection in a Pediatric Patient. Translational Pediatrics 6(2): 102-103.

3. Skelton WP, Taylor Z, Hsu J (2017) A Rare Case of Raoultella Planticola Urinary Tract Infection in an Immunocompromised Patient with Multiple Myeloma. IDCases 8: 9-11.

4. Yoon JH, Ahn YH, Chun JI, Park HJ, Park BK (2015) Acute Raoultella Planticola Cystitis in a Child with Rhabdomyosarcoma of the Bladder Neck. Pediatrics Int 57(5): 985-987.

5. Bagley ST (1985) Habitat Association of Klebsiella Species. Infection 6(2): 52-58.

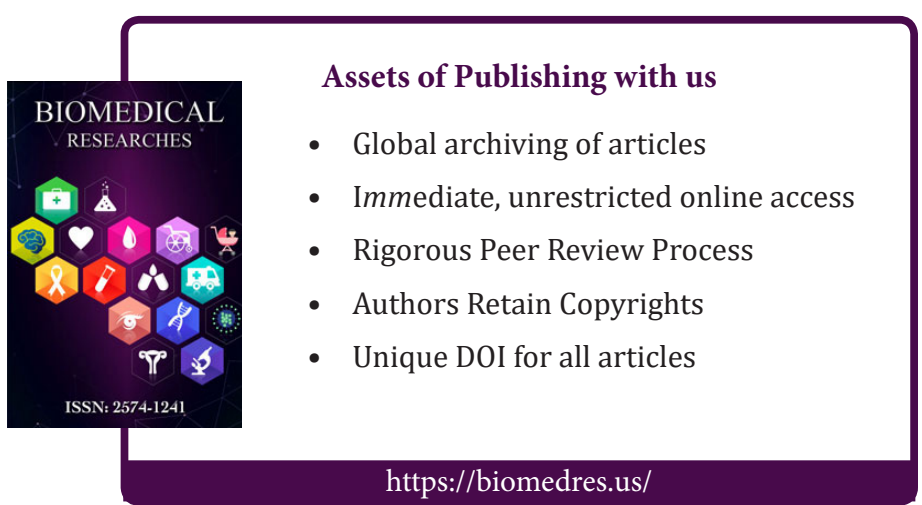

